Annales Academiæ Scientiarum Fennicæ

Mathematica

Volumen 35, 2010, 609-626

\title{
NON-CONFORMAL LOEWNER TYPE ESTIMATES FOR MODULUS OF CURVE FAMILIES
}

\author{
Tomasz Adamowicz and Nageswari Shanmugalingam \\ University of Cincinnati, Department of Mathematical Sciences \\ P.O. Box 210025, Cincinnati, OH 45221-0025, U.S.A.; tadamowi@gmail.com \\ University of Cincinnati, Department of Mathematical Sciences \\ P.O. Box 210025, Cincinnati, OH 45221-0025, U.S.A.; nages@math.uc.edu
}

\begin{abstract}
We develop various upper and lower estimates for $p$-modulus of curve families on ring domains in the setting of abstract metric measure spaces and derive $p$-Loewner type estimates for continua. These estimates are obtained for doubling metric measure spaces or $Q$-Ahlfors regular metric measure spaces supporting $(1, p)$-Poincaré inequality for the situations of $1 \leq p \leq Q$ and $p>Q$. We also study $p$-modulus estimates with respect to Riesz potentials.
\end{abstract}

\section{Introduction and preliminaries}

Recently there has been increasing interest in the geometry of the $p$-harmonic world. If $p=n$, the relations between $n$-harmonics and conformal and quasiconformal maps allow us to discover a variety of properties of $n$-harmonic functions and mappings (see e.g. Chapters 14, 15 in [HKM] or [MV1, MV2] and references therein). The situation changes when $p \neq n$. In such a case either new methods and approaches have to be developed or when applying the existing methods one has to carefully analyze the steps of reasoning. Unfortunately, the use of quasiconformal mappings as tools in the study of the $p$-modulus provides us mainly with qualitative results. Thus, the cases where the precise estimates can be obtained are worth contemplation. That is the main purpose of this note. We extend the well known classical results of Gehring [Ge] and Väisälä [Vä] on the $n$-modulus of the family of curves to the setting of $p$-modulus considered in abstract metric measure spaces.

Additional motivation for our studies comes from the problem of generalizing the notion of prime ends to the setting of $p$-modulus. To accomplish this goal the deeper understanding of $p$-modulus is necessary (see [Nä, Oh] for more on prime ends).

The paper is organized as follows. In this section we give the needed definitions, state the key technical lemmas and explain the notation. In Section 2 we present various known results about lower and upper estimates for the $p$-modulus of curves in $\overline{\mathbf{R}}^{n}$ from Väisälä's book [Vä] and Caraman's papers [C1, C2, C3, C4] to conclude with a Loewner type theorem for $p$-modulus. Section 3 is devoted to studying the upper estimates for $p$-modulus in the abstract setting of metric measure spaces. In Section 4 we develop the lower estimates in the same setting. In Section 5 we discuss the $p$-modulus in relation to Riesz potentials.

The ring domains will be for us the fundamental category of sets to deal with. An important point is that the modulus estimates for ring domains even in the Euclidean

doi:10.5186/aasfm.2010.3538

2000 Mathematics Subject Classification: Primary 30C65, 28A75, 28A78, 31C15, 46E35.

Key words: $p$-modulus of curve family, Loewner type theorem, metric measure spaces, conformal mappings, $p$-capacity, $p$-harmonic functions. 
setting are not so widely known for $p>n-1$. Also, our discussion emphasizes the role of the borderline value $p=n-1$ : for $p \leq n-1$ the curves have $p$-capacity zero and therefore the modulus analysis does not result in a viable theory.

We start by recalling selected definitions and notation commonly appearing in analysis on metric spaces. Given a metric measure space $(X, d, \mu)$, with $\mu$ a locally finite Borel measure supported on $X$, and $\Gamma$ a family of rectifiable curves in $X$, by $F(\Gamma)$ we denote the collection of all non-negative Borel measurable functions $\rho$ on $X$ such that $\int_{\gamma} \rho d s \geq 1$ for every $\gamma \in \Gamma$. The $p$-modulus of the family $\Gamma$, denoted $M_{p}(\Gamma)$, is the number

$$
M_{p}(\Gamma):=\inf _{\rho \in F(\Gamma)} \int_{X} \rho^{p} d \mu .
$$

Should $F(\Gamma)$ be empty, we set $M_{p}(\Gamma)=\infty$.

One of the fundamental concepts of analysis on metric spaces is that of the upper gradient (see [He, HK]). Following [He, Section 7.22] we say that a Borel function $\varrho: X \rightarrow[0, \infty]$ is an upper gradient of a function $u: X \rightarrow \mathbf{R}$ if for all rectifiable curves $\gamma$

$$
|u(x)-u(y)| \leq \int_{\gamma} \varrho d s,
$$

where $x$ and $y$ denote the endpoints of $\gamma$. Observe that $\varrho \equiv \infty$ is the upper gradient of every function on $X$ and if there are no rectifiable curves in $X$ then $\varrho \equiv 0$ is an upper gradient of every function on $X$. If condition (1.1) holds except for a fixed family of curves with zero $p$-modulus, then $\varrho$ is called a $p$-weak upper gradient.

We say that a metric measure space $X$ supports a $(1, p)$-Poincaré inequality if there are constants $C>0$ and $\tau \geq 1$ so that for each ball $B \subset X$ and each function $u: X \rightarrow \mathbf{R}$ and every upper gradient $\varrho$ of $u$ the following inequality holds:

$$
f_{B}\left|u-u_{B}\right| \leq C(\operatorname{diam}(B))\left(f_{\tau B} \varrho^{p}\right)^{\frac{1}{p}}
$$

where $u_{B}$ denotes the mean value of $u$ over $B$, that is $u_{B}=f_{B} u=\frac{1}{\mu(B)} \int_{B} u$. If a metric space $X$ supports $(1, p)$-Poincaré inequality, then $X$ has plenty of rectifiable curves analogous to the Loewner property for the $p$-modulus. For instance the Euclidean space $\mathbf{R}^{n}$ supports a Poincaré inequality for any $1 \leq p<\infty$, while the snowflake spaces do not support such inequality for any $p$. A doubling space (see below), supporting Poincaré inequality enables us to establish a first order calculus (see e.g. [He, HK]).

Given a set $\Omega \subset X$ we will denote its complement in $X$ by $\Omega^{c}$. For nonempty sets $E, F, K$ in $X, \Gamma=\Delta(E, F, K)$ will denote the family of rectifiable curves from $E$ to $F$ in $K$. More precisely, $\Delta(E, F, K)$ consists of compact curves $\gamma$ with one endpoint in $E$, the other in $F$, and $\gamma \backslash(E \cup F) \subset K$. As in [Vä], the modulus of the curve family $M_{p}(\Delta(E, F, K))$ is the number

$$
M_{p}(\Delta(E, F, K))=M_{p}\left(\left\{\left.\gamma\right|_{K}: \gamma \in \Delta(E, F, K)\right\}\right) .
$$

In what follows we will also appeal to the notion of $p$-capacity and its relation to $p$-modulus. Let $1<p<\infty$ and let the triple $(E, F, K)$ be as above. Consider the family of functions $u$ such that $\left.u\right|_{E} \geq 1$ and $\left.u\right|_{F} \leq 0$. Following [He] we define the 
$p$-capacity of the triple $(E, F, K)$ as

$$
\operatorname{Cap}_{p}(E, F, K)=\inf _{\varrho}\|\varrho\|_{L^{p}(K)}^{p},
$$

where the infimum is taken over the set of non-negative Borel-measurable functions $\varrho$ that are upper gradients (or weak upper gradients) of some function $u$ as above. One can obtain various capacities by considering different degrees of regularity for functions $u$, e.g. by requiring $u$ to be continuous, locally Lipshitz. With self-explanatory notation it is immediate that

$$
M_{p}(\Delta(E, F, K)) \leq \operatorname{Cap}_{p}(E, F, K) \leq \operatorname{Cont}_{-C_{a p}}(E, F, K) \leq \operatorname{locLip}_{-C_{a p}}(E, F, K) .
$$

As a matter of fact a stronger result is true. Namely, the following theorem provides us with the sufficient conditions for equalities to hold above.

Theorem 1.1. (Theorem $1.1 \mathrm{in}[\mathrm{KSh}]$ ) If $X$ is a proper $\phi$-convex metric measure space equipped with a doubling measure and supporting $(1, p)$-Poincaré inequality with $1<p<\infty$, and $\Omega$ is a domain in $X$, then for all disjoint non-empty subsets $E$ and $F$ of $\Omega$,

$$
\mathrm{M}_{p}(\Delta(E, F, \Omega))=\operatorname{Cap}_{p}(E, F, \Omega)=\operatorname{Cont}_{-C_{p a p}}(E, F, \Omega)=\operatorname{locLip}_{-C_{a p}}(E, F, \Omega) .
$$

(The first equality holds for all compact metric spaces, [HK, Proposition 2.17]).

The assumption on $\phi$-convexity can be easily bypassed by the observation that a complete doubling metric measure space supporting $(1, p)$-Poincaré inequality is quasiconvex (a result due to Semmes, see also [Ko] and references therein).

The sphere in $\mathbf{R}^{n}$, of radius $r>0$ and centered at $x_{0}$, is denoted $S=S(r)=$ $S^{n-1}\left(x_{0}, r\right)$, and $\omega_{n}=m_{n}(S)$ denotes the Lebesgue measure of $S(1)$. Suppose that $\Gamma$ is a curve family in $S(r)$. Following Section 10.1 in [Vä] we denote by $M_{p}^{S}$ the $p$ modulus of $\Gamma$ with respect to the metric space $S$ equipped with the $(n-1)$-dimensional Lebesgue measure $d m_{n-1}$. Let $\Gamma$ be a family of curves in $D \subset \overline{\mathbf{R}}^{n}$ and let $f: D \rightarrow \overline{\mathbf{R}^{n}}$ be continuous. Then $f \Gamma:=\{f \circ \gamma: \gamma \in \Gamma\}$. Consider $f_{k}(x)=k x$ for $k>0$ and $f_{k}(\infty)=\infty$. In what follows we will appeal to

Proposition 1.2. (Theorem 8.2 in [Vä]) With $k \Gamma:=f_{k} \Gamma$, we have $M_{p}(k \Gamma)=$ $k^{n-p} M_{p}(\Gamma)$.

Remark 1.3. Proposition 1.2 holds also for the spherical modulus $M_{p}^{S}$ with $n$ replaced by $n-1$, the natural dimension of sphere $S$. More specifically, if $\Gamma$ is a family of curves in $S$ and $F_{k}=\left.f_{k}\right|_{S}$, then

$$
M_{p}^{F_{k}(S)}\left(F_{k} \Gamma\right)=k^{n-1-p} M_{p}^{S}(\Gamma) .
$$

In what follows we will need to know the $p$-modulus of the family of non-constant rectifiable curves passing through a given point. The result stating that it is zero is known for $p=n$ (see [Vä, Section 7.9]). Below we discuss the counterpart of this result for the general doubling metric measure spaces with $p \geq 1$. We also provide upper and lower estimates for $M_{p}$.

We say that a metric measure space is doubling if $\mu$ is a Borel regular measure on the metric space $(X, d)$ and there exists $C>0$ such that for all $x \in X$ and every $r>0$

$$
\mu(B(x, 2 r)) \leq C \mu(B(x, r))
$$


As a consequence of the doubling property, if in addition $X$ is path connected, then there exist constants $Q_{1} \geq Q_{2} \geq 1$ and $C \geq 1$ such that for all points $x_{0}$ and $0<r \leq R<\operatorname{diam}(\mathrm{X})$

$$
\frac{1}{C}\left(\frac{r}{R}\right)^{Q_{1}} \leq \frac{\mu\left(B\left(x_{0}, r\right)\right)}{\mu\left(B\left(x_{0}, R\right)\right)} \leq C\left(\frac{r}{R}\right)^{Q_{2}} .
$$

Before we proceed, let us recall the following (Definition 6.3 in [Vä]).

Definition 1.4. Let $\Gamma_{1}$ and $\Gamma_{2}$ be curve families in $X$. We say that $\Gamma_{2}$ is $m i$ norized by $\Gamma_{1}$ and denote $\Gamma_{1}<\Gamma_{2}$ if every $\gamma \in \Gamma_{2}$ has a subcurve in $\Gamma_{1}$.

It can be seen directly from the definition of $p$-modulus that if $\Gamma_{1}<\Gamma_{2}$, then $M_{p}\left(\Gamma_{2}\right) \leq M_{p}\left(\Gamma_{1}\right)$. Let us also recall some other concepts and results from the metric measure space theory that will be needed in further discussion. Given $x$ and $y$ in $X$ we denote by $\Gamma_{x y}$ the family of compact rectifiable curves in $X$ joining $x$ and $y$. To show the measurability of certain functions in Proposition 4.1 and Theorem 5.1 we will appeal to the following result.

Proposition 1.5. (Corollary 1.10 in [JJRRS]) Let $X$ be a complete separable metric space equipped with a $\sigma$-finite Borel measure $\mu$, and let $\varrho: X \rightarrow[0, \infty]$ be a Borel function. Then for each $x_{0} \in X$, the function $u: X \rightarrow[0, \infty]$, defined for all $x \in X$ by

$$
u(x)=\inf \left\{\int_{\gamma} \varrho d s: \gamma \in \Gamma_{x_{0} x}\right\}
$$

is measurable with respect to the $\sigma$-algebra generated by analytic sets, and therefore, it is $\mu$-measurable.

\section{2. $p$-Modulus estimates in $\mathbf{R}^{n}$}

The purpose of this section is to recall upper and lower ( $p$-Loewner type) estimates in $\overline{\mathbf{R}^{n}}$. Our goal is also to present known results in Euclidean setting for the purpose of comparing and contrasting with the situation in more general metric measure spaces.

In order to obtain upper bounds for the $p$-modulus it suffices to consider one choice of admissible function $\varrho \in F(\Gamma)$. Such estimates in $\overline{\mathbf{R}}^{n}$ are nowadays classical (see e.g. 2.11 in $[\mathrm{HKM}]$ ). However, to get a lower bound one needs to consider all admissible functions in $F(\Gamma)$, and hence lower bounds are more difficult to obtain.

We recall the following result proved by Caraman.

Theorem 2.1 (Theorem 3 in [C1]). Let $n \geq 2, n-1<p$ and let $E$ and $F$ be nonempty nonintersecting subsets of the sphere $S=S^{n-1}\left(x_{0}, r\right)$. Consider the curve family $\Gamma=\Delta(E, F, S)$. Then

$$
M_{p}^{S}(\Gamma) \geq \frac{c_{p}^{n}}{r^{p-n+1}}
$$

The equality holds when $E=\{a\}$ and $F=\{b\}$ with $a$ and $b$ antipodal points on $S$.

Before stating the next result let us recall the definition of a ring (see Section 11 in $[\mathrm{Vä}])$.

Definition 2.2. A domain $A$ in $\overline{\mathbf{R}}^{n}$ is called a ring if $A^{c}$ has exactly two components. 
In what follows components of the complement of a ring will be denoted by $C_{0}, C_{1}$ and the ring by $R\left(C_{0}, C_{1}\right)$. The next lemma is a direct consequence of Theorem 2.1 and is used to investigate the $p$-modulus on the ring domains.

Lemma 2.3. (Theorem 4 in [C1]) Suppose that $0<a<b$ and that $E$ and $F$ are disjoint sets in $\overline{\mathbf{R}}^{n}$ such that every sphere $S^{n-1}(t)$ for $a<t<b$ meets both $E$ and $F$. If $G$ contains the spherical ring $A=B^{n}(b) \backslash \bar{B}^{n}(a)$ and if $\Gamma=\Delta(E, F, G)$, then for $n-1<p<n$ or $p>n$,

$$
M_{p}(\Gamma) \geq \frac{c_{p}^{n}}{n-p}\left(b^{n-p}-a^{n-p}\right),
$$

where $c_{p}^{n}$ is the constant in Theorem 2.1. The equality holds if $G=A$ and $E$ and $F$ are the components of $L \cap A$ for $L$ being a line through 0 .

In order to further analyze the $p$-modulus of ring domains we introduce the following family of rings (compare with Definition 11.6 in [Vä]). For positive $r$ and $R$, let

$$
\begin{gathered}
\Phi_{n}(r, R)=\left\{A=R\left(C_{0}, C_{1}\right) \subset \overline{\mathbf{R}}^{n}: 0 \in C_{0}, \exists a \in C_{0} \text { with }|a|=R,\right. \\
\left.\infty \in C_{1}, \exists b \in C_{1} \text { with }|b|=r\right\} .
\end{gathered}
$$

For a ring $A=R\left(C_{0}, C_{1}\right)$ we set $\Gamma_{A}=\Delta\left(C_{0} \cap \bar{A}, C_{1} \cap \bar{A}, A\right)$. We are in a position to define

$$
H_{p}(r, R):=\inf _{A \in \Phi(r, R)} M_{p}\left(\Gamma_{A}\right) .
$$

Note that by Proposition $1.2, \frac{1}{R^{n-p}} H_{p}(r, R)=H_{p}\left(\frac{r}{R}, 1\right)$. For this reason it suffices to consider

$$
H_{p}(r):=H_{p}(r, 1) \text { and } \Phi_{n}(r):=\Phi_{n}(r, 1) .
$$

Theorem 2.4. (Theorem 8 in $[\mathrm{C} 2])$ The function $H_{p}(r)$ has the following properties.

(1) $H_{p}$ is a decreasing function,

(2) $\lim _{r \rightarrow \infty} H_{p}(r)=0$ for $p \geq n$, while $\lim _{r \rightarrow \infty} H_{p}(r)=\omega_{n-1}\left(\frac{|n-p|}{p-1}\right)^{p-1}$ for $p<n$; moreover $H_{p}(r)<\infty$ for all $r>0$,

(3) $\lim _{r \rightarrow 0} H_{p}(r)=\infty$ for $p \geq n$, while $\lim _{r \rightarrow 0} H_{p}(r) \geq \frac{c_{p}^{n}}{n-p}>0$ for $n-1<p<n$ with $c_{p}^{n}$ as in Theorem 2.1,

(4) $H_{p}(r)>0$ for $p>n-1$ and for all $r>0$.

Remark 2.5. One may expect that in the general setting of metric measure spaces the lower bound for $H_{p}$ in (4) above follows from a $(1, p)$-Poincaré inequality.

The optimality in cases (2) and (3) for $p<n$ is demonstrated by the following examples.

Example 2.6. By Corollary 1 in [C3] we have that

$$
\lim _{r \rightarrow \infty} M_{p}\left(\Gamma_{A_{G}(r)}\right) \leq \omega_{n-1}\left(\frac{|n-p|}{p-1}\right)^{p-1},
$$

where for $r>1, \Gamma_{A_{G}(r)}=\Delta\left(\overline{B(0,1)},\left\{x: r \leq x_{1}<\infty, x_{2}=\ldots=x_{n}=0\right\}, \mathbf{R}^{n}\right)$ represents the path family corresponding to Grötzsch ring. The optimality of case (2) for $p<n$ now follows immediately from $H_{p}(r) \leq M_{p}\left(\Gamma_{A_{G}(r)}\right)$. 
A weak optimality in the case (3) for $p<n$, that is $\lim _{r \rightarrow 0} H_{p}(r)<\infty$, is a consequence of the following example.

Example 2.7. Fix $0<r<1, n=2$, and let $E=[-1,0], F=[r, \infty]$. Then $R(E, F) \in \Phi_{2}(r)$, and so $H_{p}(r) \leq M_{p}\left(\Delta\left(E, F, \mathbf{R}^{2}\right)\right)$. Let $\Gamma_{r}$ be the collection of all rectifiable curves connecting $E$ to $F$ in the disc $B(0,2)$, and $\Gamma_{1}$ be the collection of all rectifiable curves connecting the circle $S(0,1)$ to the circle $S(0,2)$. Then $\Gamma_{r} \subset$ $\Delta\left(E, F, \mathbf{R}^{2}\right)$, and $\Gamma_{1}<\Delta\left(E, F, \mathbf{R}^{2}\right) \backslash \Gamma_{r}$. It follows that

$$
M_{p}\left(\Delta\left(E, F, \mathbf{R}^{2}\right)\right) \leq M_{p}\left(\Gamma_{r}\right)+M_{p}\left(\Gamma_{1}\right) .
$$

Since the function $\rho(z)=(\pi|z|)^{-1} \chi_{B(0,2)}(z)$ is in $F\left(\Gamma_{r}\right)$, we have when $p<2$,

$$
M_{p}\left(\Gamma_{r}\right) \leq \frac{2 \pi^{1-p}}{2-p} \frac{1-r^{2-p}}{(1-r)^{2-p}} .
$$

Since every curve in $\Gamma_{1}$ has length at least 1 , we also have

$$
M_{p}\left(\Gamma_{1}\right) \leq|B(0,2) \backslash B(0,1)|=3 \pi \text {. }
$$

It follows that

$$
H_{p}(r) \leq \frac{2 \pi^{1-p}}{2-p} \frac{1-r^{2-p}}{(1-r)^{2-p}}+3 \pi
$$

Hence when $p<2$ we have $\lim _{r \rightarrow 0} H_{p}(r) \leq \frac{2 \pi^{1-p}}{2-p}+3 \pi<\infty$.

In the next observation we express the lower bound for the $p$-modulus in terms of $H_{p}$ (compare to Theorem 11.9 in [Vä] for the case of $n$-modulus).

Proposition 2.8. (Theorem 9 in [C2]) Let $A=R\left(C_{0}, C_{1}\right)$ be a ring such that $a, b \in C_{0}$ and $c, \infty \in C_{1}$. Then

$$
M_{p}\left(\Gamma_{A}\right) \geq H_{p}\left(\frac{|c-a|}{|b-a|}\right)|b-a|^{n-p}, \quad \text { for } p>1 .
$$

The above proposition together with Theorem 3.1 result in the following characterization of rings with zero $p$-modulus (the proof in the Euclidean setting is similar to the case $p=n$, Theorem 11.0 in [Vä]).

Proposition 2.9. Let $A=R\left(C_{0}, C_{1}\right)$ be a ring. Then if $n-1<p \leq n$,

$$
M_{p}\left(\Gamma_{A}\right)=0 \quad \Longleftrightarrow \quad \# C_{0}=1 \text { or } \# C_{1}=1 \text {. }
$$

\section{Upper $p$-modulus estimates in the abstract setting}

Recall from Section 1 (see $(\star)$ ) that one of the consequences of the doubling property for path connected spaces $X$, is that there exist constants $Q_{1} \geq Q_{2} \geq 1$ and $C \geq 1$ such that for all points $x_{0}$ and $0<r \leq R<\operatorname{diam}(\mathrm{X})$,

$$
\frac{1}{C}\left(\frac{r}{R}\right)^{Q_{1}} \leq \frac{\mu\left(B\left(x_{0}, r\right)\right)}{\mu\left(B\left(x_{0}, R\right)\right)} \leq C\left(\frac{r}{R}\right)^{Q_{2}} .
$$

Theorem 3.1. Let $X$ be a path connected doubling space with $Q_{2}>1$. Let $x_{0} \in X$ and $\Gamma$ be a collection of non-constant paths that pass through $x_{0}$. If $1 \leq p \leq$ $Q_{2}$, then $M_{p}(\Gamma)=0$.

Let $0<2 r<R$ and denote by

$$
\Gamma(r, R)=\Delta\left(\bar{B}\left(x_{0}, r\right), X \backslash B\left(x_{0}, R\right), B\left(x_{0}, R\right)\right)
$$


the family of curves joining $\bar{B}\left(x_{0}, r\right)$ and $X \backslash B\left(x_{0}, R\right)$. If $1 \leq p<Q_{2}$, we have the upper estimate

$$
M_{p}(\Gamma) \leq C\left(R_{0}, Q_{1}\right) \frac{C_{R, \frac{r}{4}}}{C_{R, 2 r}^{p}} R^{Q_{1}-Q_{2}},
$$

with $C_{R, r}=\frac{1}{2^{\alpha}-2}\left(\frac{1}{r^{\alpha-1}}-\frac{1}{R^{\alpha-1}}\right), \alpha=\frac{Q_{2}-1}{p-1}$ and constant $R_{0}>0$ such that $r<R_{0}<R$. If $p=Q_{2}$, the corresponding estimate reads

$$
M_{Q_{2}}(\Gamma) \leq C^{\prime}\left(R_{0}, Q_{1}\right) \frac{R^{Q_{1}-Q_{2}}}{C_{R, r}^{Q_{2}-1}}, \text { where } C_{R, r}=\ln \left(\frac{R}{r}\right) .
$$

In the Euclidean setting $Q_{1}=Q_{2}=n$ and we retrieve the $p$-capacity estimates for the Euclidean annuli (see for example [HKM, Lemma 2.11]).

Proof. We will first apply the telescoping argument to estimate the modulus of $\Gamma(r, R)$ and then obtain the first part of the lemma by letting $r \rightarrow 0$. The proof is divided into two cases. First, let $p<Q_{2}$. Consider

$$
\varrho(x)=\frac{1}{C_{R, 2 r}} \frac{1}{d\left(x_{0}, x\right)^{\alpha}}, \quad \alpha=\frac{Q_{2}-1}{p-1}>1,
$$

where $C_{R, 2 r}=\frac{1}{2^{\alpha}-2}\left(\frac{1}{(2 r)^{\alpha-1}}-\frac{1}{R^{\alpha-1}}\right)$. Choose $k_{0}$ to be the smallest positive integer such that

$$
2^{-k_{0}} R \leq r<2^{1-k_{0}} R
$$

Divide the annulus into dyadic annular regions $\mathscr{R}_{i}=B\left(x_{0}, 2^{-i} R\right) \backslash B\left(x_{0}, 2^{-i-1} R\right)$ with radii $2^{-i-1} R<2^{-i} R$, for $i=0, \ldots, k_{0}-1$. Consider any curve $\gamma \in \Gamma(r, R)$ and denote by $\gamma_{i}$ the part of $\gamma$ in the $i$-th subring $\mathscr{R}_{i}$ (note that $\gamma_{i}$ might be disconnected consisting of a family of arcs). Let $\beta_{i}$ be a subarc of $\gamma_{i}$ connecting the inner sphere of $\mathscr{R}_{i}$ with its outer sphere. Next, we show that $\varrho$ is an admissible function for $\Gamma(r, R)$.

$$
\begin{aligned}
\int_{\gamma} \varrho d s & =\sum_{i=0}^{k_{0}} \int_{\gamma_{i}} \varrho d s \geq \sum_{i=0}^{k_{0}-1} \int_{\beta_{i}} \varrho d s \geq \sum_{i=0}^{k_{0}-1} \frac{l\left(\beta_{i}\right)}{C_{R, 2 r}\left(2^{-i} R\right)^{\alpha}} \\
& \geq \frac{1}{2} \sum_{i=0}^{k_{0}-1} \frac{1}{C_{R, 2 r}\left(2^{-i} R\right)^{\alpha-1}}=\frac{1}{2 C_{R, 2 r} R^{\alpha-1}} \sum_{i=0}^{k_{0}-1} 2^{i(\alpha-1)} \\
& =\frac{1}{2 C_{R, 2 r} R^{\alpha-1}} \frac{2^{(\alpha-1)\left(k_{0}-1\right)}-1}{2^{\alpha-1}-1} \geq \frac{1}{C_{R, 2 r}\left(2^{\alpha}-2\right) R^{\alpha-1}}\left(\left(\frac{R}{2 r}\right)^{\alpha-1}-1\right) \\
& \geq \frac{1}{C_{R, 2 r}\left(2^{\alpha}-2\right)}\left(\frac{1}{(2 r)^{\alpha-1}}-\frac{1}{R^{\alpha-1}}\right)=1,
\end{aligned}
$$

and hence $\varrho \in F(\Gamma(r, R))$. Below the constant $C_{R, r}$ may change from line to line, but always has the form $C_{R, r}=C\left(\frac{1}{r^{\alpha-1}}-\frac{1}{R^{\alpha-1}}\right)$, for $C$ depending on $\alpha, p, Q_{2}$ and the doubling constant in $(\star)$. The following chain of inequalities leads us to the upper estimate for $M_{p}(\Gamma(r, R))$.

$$
M_{p}(\Gamma(r, R)) \leq \int_{B\left(x_{0}, R\right) \backslash B\left(x_{0}, r\right)} \varrho^{p} d \mu=\sum_{i=0}^{k_{0}-1} \int_{B\left(x_{0}, 2^{-i} R\right) \backslash B\left(x_{0}, 2^{-i-1} R\right)} \varrho^{p} d \mu
$$




$$
\begin{aligned}
& \leq \sum_{i=0}^{k_{0}-1} \frac{\mu\left(B\left(x_{0}, 2^{-i} R\right)\right)}{C_{R, 2 r}^{p}} \frac{1}{\left(2^{-(i+1)} R\right)^{\alpha p}} \\
& \leq \frac{2^{\alpha p}}{C_{R, 2 r}^{p}} \frac{\mu\left(B\left(x_{0}, R\right)\right)}{R^{\alpha p}} \sum_{i=0}^{k_{0}-1} 2^{-i\left(Q_{2}-\alpha p\right)} \quad(\text { by }(\star)) \\
& \leq \frac{1}{C_{R, 2 r}^{p}} \frac{\mu\left(B\left(x_{0}, R\right)\right)}{R^{\alpha p}} \frac{2^{\left(\alpha p-Q_{2}\right)\left(k_{0}+1\right)}-1}{2^{\alpha p-Q_{2}}-1} \\
& \leq \frac{1}{C_{R, 2 r}^{p}} \frac{\mu\left(B\left(x_{0}, R\right)\right)}{R^{\alpha p}}\left(4^{\alpha p-Q_{2}}\left(\frac{R}{r}\right)^{\alpha p-Q_{2}}-1\right) \\
& \leq \frac{1}{C_{R, 2 r}^{p}} \frac{\mu\left(B\left(x_{0}, R\right)\right)}{R^{Q_{2}}}\left(\left(\frac{4}{r}\right)^{\alpha p-Q_{2}}-\frac{1}{R^{\alpha p-Q_{2}}}\right) \\
& \leq \frac{C_{R, \frac{r}{4}}}{C_{R, 2 r}^{p}} \frac{\mu\left(B\left(x_{0}, R\right)\right)}{R^{Q_{2}}}, \quad\left(\text { as } \alpha p-Q_{2}=\alpha-1 .\right)
\end{aligned}
$$

By $(\star)$ again,

$$
M_{p}(\Gamma(r, R)) \leq \frac{C_{R, \frac{r}{4}}}{C_{R, 2 r}^{p}} \frac{\mu\left(B\left(x_{0}, R_{0}\right)\right)}{R_{0}^{Q_{1}}} R^{Q_{1}-Q_{2}} .
$$

Now let $p=Q_{2}$ and hence $\alpha=1$. Computations similar to these at (3.3) result in

$$
\int_{\gamma} \varrho d s \geq \sum_{i=0}^{k_{0}} \frac{l\left(\beta_{i}\right)}{C_{R, r} 2^{-i} R} \geq \sum_{i=0}^{k_{0}} \frac{1}{C_{R, r}}=\frac{k_{0}}{C_{R, r}} \geq 1,
$$

for a suitable choice of constant $C$ in $C_{R, r}=C \ln \left(\frac{R}{r}\right)$. By the reasoning analogous to (3.4) we arrive at the following estimate:

$$
\begin{aligned}
\int_{B\left(x_{0}, R\right) \backslash B\left(x_{0}, r\right)} \varrho^{p} d \mu & \leq \frac{\mu\left(B\left(x_{0}, R\right)\right)}{C_{R, r}^{p}} \sum_{i=0}^{k_{0}} \frac{2^{-i Q_{2}}}{\left(2^{-i} R\right)^{\alpha p}} \quad \text { (by doubling condition) } \\
& \leq \frac{\mu\left(B\left(x_{0}, R\right)\right)}{C_{R, r}^{p} R^{\alpha p}} \sum_{i=0}^{k_{0}} 2^{-i\left(Q_{2}-\alpha p\right)} \leq \frac{\mu\left(B\left(x_{0}, R\right)\right)}{C_{R, r}^{p} R^{Q_{2}}} k_{0} \\
& \leq \frac{\mu\left(B\left(x_{0}, R\right)\right)}{R^{Q_{2}}} \frac{1}{C_{R, r}^{p}} \ln \left(\frac{R}{r}\right) \leq \frac{\mu\left(B\left(x_{0}, R_{0}\right)\right)}{R_{0}{ }^{Q_{1}}} \frac{R^{Q_{1}-Q_{2}}}{C_{R, r}^{Q_{2}-1}}
\end{aligned}
$$

Note that if $Q_{1}=Q_{2}=p$, then as $R \rightarrow \infty$ the expression on the right hand side approaches 0 . On the other hand, if $Q_{1}=Q_{2}>p$, we can only conclude that

$$
\lim _{R \rightarrow \infty} M_{p}(\Gamma(r, R)) \leq C\left(R_{0}, Q_{1}\right) r^{Q_{2}-p} .
$$

Since our main goal is to estimate the modulus either for $r \rightarrow 0$ (with $r<R$ ) or for large values of $R$, we may assume without loss of generality that $R>R_{0}$ for some positive constant $R_{0}$. The above estimates for $1 \leq p<Q_{2}$ imply that

$$
M_{p}(\Gamma(r, R)) \leq \frac{C_{R, \frac{r}{4}}}{C_{R, 2 r}^{p}} \frac{\mu\left(B\left(x_{0}, R_{0}\right)\right)}{R_{0}^{Q_{1}}} R^{Q_{1}-Q_{2}} .
$$


Denote by $\Gamma(R)=\bigcap_{0<r<R} \Gamma(r, R)$. Then $\Gamma>\bigcup_{n \in \mathbf{N}} \Gamma(1 / n)$ (see Definition 1.4), that is, every curve in $\Gamma$ has a subcurve that belongs to $\bigcup_{n \in \mathbf{N}} \Gamma(1 / n)$. Hence $M_{p}(\Gamma) \leq$ $M_{p}\left(\bigcup_{n \in \mathbf{N}} \Gamma(1 / n)\right)$. Observe that

$$
\frac{C_{R, \frac{r}{4}}}{C_{R, 2 r}^{p}}=C \frac{\left(\frac{4}{r}\right)^{\alpha-1}-\frac{1}{R^{\alpha-1}}}{\left(\frac{1}{(2 r)^{\alpha-1}}-\frac{1}{R^{\alpha-1}}\right)^{p}} \rightarrow 0, \text { for } r \rightarrow 0 .
$$

Therefore, if $1 \leq p<Q_{2}$ the first part of the lemma follows immediately from the fact that

$$
M_{p}(\Gamma(R))=\lim _{r \rightarrow 0} M_{p}(\Gamma(r, R))=0, \text { and hence } M_{p}(\Gamma) \leq \sum_{n=0}^{\infty} M_{p}(\Gamma(1 / n))=0 .
$$

Similar argument allows us to handle the case $p=Q_{2}$. Namely, by (3.6) we have that

$$
\frac{1}{C_{R, r}^{p-1}}=C \frac{1}{(\ln R-\ln r)^{p-1}} \rightarrow 0, \text { for } r \rightarrow 0 .
$$

This completes the argument in the case $p=Q_{2}$.

Remark 3.2. Another type of upper estimates have been recently obtained by Garofalo and Marola, see Theorem 3.4 in [GM]. In our setting and notation this result states that for a bounded open subset $\Omega \subset X$ and $x_{0} \in \Omega$, if $0<r<R<R_{0}(\Omega)$, then

$$
\operatorname{Cap}_{p}\left(\overline{B\left(x_{0}, r\right)}, B\left(x_{0}, R\right)\right) \leq \begin{cases}C(p, \Omega) \frac{\mu\left(B\left(x_{0}, r\right)\right)}{r^{p}}, & 1<p<Q_{2}, \\ C(p, \Omega) \frac{\mu\left(B\left(x_{0}, r\right)\right)}{r^{p}}\left(\ln \frac{R}{r}\right)^{1-Q_{2}}, & p=Q_{2} .\end{cases}
$$

These estimates are not equivalent to ours. For instance if $p \approx Q_{2}$, the above inequalities give the upper bound for $M_{p} \approx C$ while we have the upper estimate for $M_{p} \approx R^{Q_{1}-Q_{2}}$. Similar discrepancies appear when $p=Q_{2}$. For example, if space $X$ is $Q_{2}$-Ahlfors regular, then for small $R$ our estimate is stronger.

Remark 3.3. If $Q_{1}=Q_{2}$, that is, if $X$ is locally Ahlfors regular, then as a byproduct of the computations (3.4), we get the upper bound for the $p$-modulus for large values of $R$ :

$$
M_{p}(\Gamma(r, R)) \leq \frac{C_{R, \frac{r}{4}}}{C_{R, 2 r}^{p}} \frac{\mu\left(B\left(x_{0}, R_{0}\right)\right)}{R_{0}^{Q_{1}}} \rightarrow C \frac{4^{\alpha p-Q_{2}}}{r^{\left(\alpha p-Q_{2}\right)(p-1)}}=C\left(p, Q_{2}\right) r^{Q_{2}-p},
$$

for $R \rightarrow \infty$.

Remark 3.4. The above arguments allow us to prove part (2) of Theorem 2.4 without appealing to Euclidean techniques. Namely, using the discussion of (3.8), Remark 3.3 and estimate (3.6) with $R_{0}=1, R=1+r$ and $r=1$ we arrive at the following estimates:

$$
\begin{aligned}
& p<Q_{2}=Q_{1}=n: H_{p}(r) \leq M_{p}(\Gamma(1,1+r)) \leq \frac{C_{1+r, \frac{1}{4}}}{C_{1+r, 2}} \\
& \leq C \frac{4^{\alpha-1}-\frac{1}{(1+r)^{\alpha-1}}}{\left(\frac{1}{2}-\frac{1}{(1+r)^{\alpha-1}}\right)^{p}} \rightarrow C(p, n), \text { for } r \rightarrow \infty, \\
& p=Q_{2}=Q_{1}=n: H_{p}(r) \leq \frac{C(n)}{(\ln (1+r))^{Q_{2}-1}} \rightarrow 0, \text { for } r \rightarrow \infty .
\end{aligned}
$$


However, in this general non-Euclidean setting, we do not have explicit sharp expressions for the constants $C(p, n), C(n)$.

\section{Lower $p$-modulus estimates in the abstract setting}

The purpose of this section is to discuss the counterparts of Theorem 2.1 and Lemma 2.3 in the abstract setting. The essence of the calculations below reveals that a viable theory of $p$-Loewner spaces can be successfully developed beyond the Euclidean setting. The explicit expressions for the constants involved in the estimates however are lacking in the metric setting. Our approach is very much in the spirit of Heinonen-Koskela [HK].

Let $X$ be a metric measure space supporting a $(1, p)$-Poincaré inequality (see e.g. Chapter 4 in $[\mathrm{He}]$ for information on Poincaré inequality). Assume that the following Ahlfors regularity type condition holds: for every ball $B_{R}$ in $X$ of radius $R<\operatorname{diam} X$,

$$
\frac{1}{C} R^{Q} \leq \mu(B(R)) \leq C R^{Q},
$$

with $Q \geq 1$ and $C \geq 1$. Note that (4.1) implies the previously discussed condition $(\star)$. For distinct points $x, y \in X$ denote by $\Gamma_{x y}$ the collection of all compact rectifiable curves in $X$ connecting $x$ to $y$. For $k \in \mathbf{N}$ such that $k>\frac{2009}{d(x, y)}$ let $\Gamma_{x y}^{k}$ denote the collection of curves in $X$ connecting $\bar{B}(x, 1 / k)$ to $\bar{B}(y, 1 / k)$.

Proposition 4.1. With the above notation, if $p>Q$, the space $X$ is $Q$-Ahlfors regular and supports a $(1, p)$-Poincaré inequality, then

$$
M_{p}\left(\Gamma_{x y}\right) \geq C d(x, y)^{Q-p} .
$$

Proof. Let $k>2009 / d(x, y)$ and $\varrho_{k} \in F\left(\Gamma_{x y}^{k}\right)$. Define a function $u_{k}: X \rightarrow \mathbf{R}$ by

$$
u_{k}(z)=\min \left\{\inf _{\substack{\gamma \text { connecting } \\ \bar{B}(x, 1 / k) \text { to } z}} \int_{\gamma} \varrho_{k} d s, 1\right\} .
$$

The same argument as in [JJRRS, Corollary 1.10] gives us that $u_{k}$ is measurable and since $\rho_{k} \in L^{p}(X)$ is an upper gradient of $u_{k}$, by the Poincaré inequality $u_{k} \in N_{l o c}^{1, p}(X)$. Note that $u_{k} \equiv 1$ on $\bar{B}\left(y, \frac{1}{k}\right)$, whereas $u_{k} \equiv 0$ on $\bar{B}\left(x, \frac{1}{k}\right)$.

Let $B_{0}=B(x, 2 d(x, y))$ and for $i \in \mathbf{N}$ consider the family of balls

$$
B_{i}=B\left(x, 2^{1-i} d(x, y)\right), \quad B_{-i}=B\left(y, 2^{1-i} d(x, y)\right) .
$$

Since $x$ and $y$ are Lebesgue points of $u_{k}$, we have by Ahlfors regularity and $(1, p)$ Poincaré inequality,

$$
\begin{aligned}
1=\left|u_{k}(x)-u_{k}(y)\right| & \leq \sum_{i \in \mathbf{Z}}\left|\left(u_{k}\right)_{B_{i}}-\left(u_{k}\right)_{B_{i+1}}\right| \leq C \sum_{i \in \mathbf{Z}} f_{B_{i}}\left|u_{k}-\left(u_{k}\right)_{B_{i}}\right| d \mu \\
& \leq C \sum_{i \in \mathbf{Z}} d(x, y) 2^{-|i|}\left(f_{B_{i}} \varrho_{k}^{p} d \mu\right)^{\frac{1}{p}} \\
& \leq C \sum_{i \in \mathbf{Z}}\left(d(x, y) 2^{-|i|}\right)^{1-\frac{Q}{p}}\left(\int_{X} \varrho_{k}^{p} d \mu\right)^{\frac{1}{p}} \\
& \leq C d(x, y)^{1-\frac{Q}{p}}\left(\int_{X} \varrho_{k}^{p} d \mu\right)^{\frac{1}{p}} .
\end{aligned}
$$


Therefore $\int_{X} \varrho_{k}^{p} d \mu \geq C d(x, y)^{Q-p}$ and therefore $M_{p}\left(\Gamma_{x y}^{k}\right) \geq C d(x, y)^{Q-p}$. The assumption that $p>Q$ has been used to ensure the convergence of the geometric series in (4.4). The proof is now completed by invoking Proposition 4.2 below.

Note that $\Gamma_{x y}=\bigcap_{k \in \mathbf{N}} \Gamma_{x y}^{k}$. Since $\Gamma_{x y}^{k+1} \subseteq \Gamma_{x y}^{k}$, the subadditivity of modulus implies that $M_{p}\left(\Gamma_{x y}^{1}\right) \geq M_{p}\left(\Gamma_{x y}^{2}\right) \geq \cdots \geq M_{p}\left(\Gamma_{x y}^{k}\right) \geq M_{p}\left(\Gamma_{x y}^{k+1}\right) \geq \cdots$. This together with Proposition 4.1 give us that $\lim _{k \rightarrow \infty} M_{p}\left(\Gamma_{x y}^{k}\right)$ exists and

$$
\lim _{k \rightarrow \infty} M_{p}\left(\Gamma_{x y}^{k}\right) \geq C d(x, y)^{Q-p} .
$$
Then

$$
\lim _{k \rightarrow \infty} M_{p}\left(\Gamma_{x y}^{k}\right)=M_{p}\left(\Gamma_{x y}\right) .
$$

Proof. First suppose that $\mu(X)<\infty$. Since for every $k$ we have $M_{p}\left(\Gamma_{x y}^{k}\right) \geq$ $M_{p}\left(\Gamma_{x y}\right)$, it suffices to prove that $M_{p}\left(\Gamma_{x y}\right) \geq \lim _{k \rightarrow \infty} M_{p}\left(\Gamma_{x y}^{k}\right)$. To do so, let us consider $\varrho \in F\left(\Gamma_{x y}\right)$ such that

$$
\int_{X} \varrho^{p} d \mu \leq M_{p}\left(\Gamma_{x y}\right)+\eta
$$

Since $X$ is locally compact and $\varrho \in L^{p}(X)$, the Vitali-Carathéodory Theorem implies that without loss of generality $\varrho$ can be assumed to be lower semicontinuous on $X$ (see page 57 in $[\mathrm{Ru}]$ ). Fix $\epsilon>0$ such that with $\varrho_{\epsilon}=\max \{\varrho, \epsilon\}$, we have $\int_{X} \varrho_{\epsilon}^{p} d \mu \leq$ $\int_{X} \varrho^{p} d \mu+\eta$. We will show that for all $\delta>0$, there exists $k_{\delta} \in \mathbf{N}$ such that for all $k \in \mathbf{N}$ with $k>k_{\delta}$, we have that for all curves $\gamma \in \Gamma_{x y}^{k}$,

$$
\int_{\gamma} \varrho_{\epsilon} d s \geq 1-\delta
$$

Suppose this is not true. Then there exists $0<\delta<1$ such that for all $k \in \mathbf{N}$ there is $n_{k}>k$, so that for some $\gamma_{n_{k}} \in \Gamma_{x y}^{n_{k}}$ it holds that

$$
\int_{\gamma_{n_{k}}} \varrho_{\epsilon} d s<1-\delta
$$

Since $\varrho_{\epsilon} \geq \epsilon$ on $X$,

$$
\epsilon \ell\left(\gamma_{n_{k}}\right) \leq \int_{\gamma_{n_{k}}} \varrho_{\epsilon} d s<1-\delta, \text { so } l\left(\gamma_{n_{k}}\right)<\frac{1-\delta}{\epsilon},
$$

and so for all $k, \ell\left(\gamma_{n_{k}}\right)<(1-\delta) / \epsilon$. Note that $\gamma_{n_{k}}$ connects a point in $\bar{B}\left(x, \frac{1}{n_{k}}\right)$ to a point in $\bar{B}\left(y, \frac{1}{n_{k}}\right)$. The Arzela-Ascoli theorem implies that $\gamma_{n_{k}} \rightarrow \gamma$ uniformly (on a subsequence if necessary) so that $\ell(\gamma) \leq \frac{1-\delta}{\epsilon}$ and $\gamma \in \Gamma_{x y}$. Computations similar to these on page 14 of [HK] gives us that by the lower semicontinuity of $\varrho_{\epsilon}$,

$$
\lim _{k \rightarrow \infty} \int_{\gamma_{n_{k}}} \varrho_{\epsilon} d s \geq \int_{\gamma} \varrho_{\epsilon} d s .
$$

Therefore, for the above $\gamma$ it holds that

$$
\int_{\gamma} \varrho_{\epsilon} d s \leq \lim _{k \rightarrow \infty} \int_{\gamma_{n_{k}}} \varrho_{\epsilon} d s \leq 1-\delta<1
$$


which contradicts with $\varrho \in F\left(\Gamma_{x y}\right)$. Hence condition (4.8) holds. From this we infer that $\frac{1}{1-\delta} \varrho \in F\left(\Gamma_{x y}^{k}\right)$ for $k>k_{\delta}$. Hence by $(4.7)$

$$
M_{p}\left(\Gamma_{x y}^{k}\right) \leq \frac{1}{(1-\delta)^{p}} \int_{X} \varrho_{\epsilon}^{p} d \mu \leq \frac{1}{(1-\delta)^{p}}\left(M_{p}\left(\Gamma_{x y}\right)+2 \eta\right), \quad \text { if } k>k_{\delta} .
$$

Hence

$$
\lim _{k \rightarrow \infty} M_{p}\left(\Gamma_{x y}^{k}\right) \leq \frac{1}{(1-\delta)^{p}}\left(M_{p}\left(\Gamma_{x y}\right)+2 \eta\right) .
$$

The assertion of Proposition 4.2 follows by letting $\delta \rightarrow 0$ and then $\eta \rightarrow 0$.

When $\mu(X)=\infty$, the above argument can be conducted on families of curves restricted to balls in $X$. Fixing $x_{0} \in X$, for $n \in \mathbf{N}$ let $\Gamma_{x y, n}$ be the family of curves in $\Gamma_{x y}$ that lie in the ball $B\left(x_{0}, n\right)$, and $\Gamma_{x y, n}^{k}$ the family of curves in $\Gamma_{x y}^{k}$ in $B\left(x_{0}, n\right)$; the above argument yields that $M_{p}\left(\Gamma_{x y, n}\right)=\lim _{k \rightarrow \infty} M_{p}\left(\Gamma_{x y, n}^{k}\right)$. Now an application of the fact that whenever $\left(\Gamma_{N}\right)_{N}$ is a sequence of curve families such that $\Gamma_{N} \subset \Gamma_{N+1}$, then $\lim _{N \rightarrow \infty} M_{p}\left(\Gamma_{N}\right)=M_{p}\left(\cup_{N} \Gamma_{N}\right)$ gives the desired conclusion.

Given a point $x_{0} \in X$ and $0<r<R$, we consider the curve family

$$
\Gamma(r, R)=\Delta\left(\bar{B}\left(x_{0}, r\right), X \backslash B\left(x_{0}, R\right), B\left(x_{0}, R\right)\right) .
$$

Theorem 4.3. Let $X$ be a $Q$-Ahlfors regular metric measure space supporting $(1, p)$-Poincaré inequality. Assume that $p>Q$. If $X \backslash B\left(x_{0}, 2 R\right)$ is non-empty, then

$$
M_{p}(\Gamma(r, R)) \geq \frac{1}{C} \frac{\mu\left(B\left(x_{0}, R\right)\right)}{R^{p}} \geq \frac{1}{C} R^{Q-p},
$$

with constant $C$ depending quantitatively on space $X$.

Remark 4.4. Note that this result is false for $p \leq Q$, since in such a case Theorem 3.1 gives us that $M_{p}(\Gamma(r, R)) \rightarrow 0$ for $r \rightarrow 0$.

Remark 4.5. Note that Theorem 4.3 together with Proposition 4.1 imply that $M_{p}(\Gamma)=\infty$ for $p>Q$, where $\Gamma$ stands for the family of non-constant curves passing through a given point in $X$.

Proof of Theorem 4.3. Let $\varrho \in F(\Gamma(r, R))$. Analogously to Proposition 4.1 we define $u: X \rightarrow \mathbf{R}$ by

$$
u(z)=\min \left\{\inf _{\substack{\gamma \text { connecting } \\ z \text { to } X \backslash B\left(x_{0}, R\right)}} \int_{\gamma} \varrho \mathrm{d} s, 1\right\} .
$$

The proof splits into two cases.

Case 1. There exists $x \in B\left(x_{0}, r\right)$ and $y \in B\left(x_{0}, 2 R\right) \backslash B\left(x_{0}, R\right)$ such that

$$
\left|u(x)-u_{B(x, R)}\right| \leq \frac{1}{4} \quad \text { and } \quad\left|u(y)-u_{B(y, R)}\right| \leq \frac{1}{4} .
$$

Then

$$
1=|u(x)-u(y)| \leq \frac{1}{4}+\left|u_{B(x, R)}-u_{B(y, R)}\right|+\frac{1}{4},
$$

and so

$$
\frac{1}{2} \leq\left|u_{B(x, R)}-u_{B(y, R)}\right|
$$


Therefore, by the doubling property of the measure $\mu$, and by the $(1, p)$-Poincaré inequality,

$$
1 \leq C f_{B\left(x_{0}, 10 R\right)}\left|u-u_{B\left(x_{0}, 10 R\right)}\right| \leq C R\left(f_{B\left(x_{0}, 10 R\right)} \varrho^{p} d \mu\right)^{\frac{1}{p}} .
$$

Hence

$$
\int_{X} \varrho^{p} d \mu \geq \int_{B\left(x_{0}, 10 R\right)} \varrho^{p} d \mu \geq \frac{1}{C} \frac{\mu\left(B\left(x_{0}, R\right)\right)}{R^{p}} .
$$

By assumption, $X \backslash B\left(x_{0}, 2 R\right)$ is non-empty. Because of the Poincaré inequality, $X$ is path-connected. Therefore there is a point $y \in B\left(x_{0}, 2 R\right) \backslash B\left(x_{0}, R\right)$ such that $d\left(x_{0}, y\right)=3 R / 2$. As $u=0$ on $X \backslash B\left(x_{0}, R\right)$, it follows that

$$
\left|u(y)-u_{B(y, R)}\right|=u_{B(y, R)}<1 / 4 .
$$

Hence the only remaining case is the following.

Case 2. For all $x \in B\left(x_{0}, r\right)$ we have $\left|u(x)-u_{B(x, R)}\right|>\frac{1}{4}$. As $x$ is a Lebesgue point of $u$ we have by the Poincare inequality with $B_{i}=B\left(x, 2^{1-i} R\right)$

$$
\begin{aligned}
\frac{1}{4}<\left|u(x)-u_{B(x, R)}\right| & \leq \sum_{i \in \mathbf{N}}\left|u_{B_{i}}-u_{B_{i+1}}\right| \leq C \sum_{i \in \mathbf{N}} f_{B_{i}}\left|u-u_{B_{i}}\right| d \mu \\
& \leq C \sum_{i \in \mathbf{N}} 2^{-i} R\left(f_{B_{i}} \varrho^{p} d \mu\right)^{\frac{1}{p}} \leq C \sum_{i \in \mathbf{N}}\left(2^{-i} R\right)^{1-\frac{Q}{p}}\left(\int_{B_{i}} \varrho^{p} d \mu\right)^{\frac{1}{p}} \\
& \leq C R^{1-\frac{Q}{p}} \sum_{i \in \mathbf{N}} 2^{-i\left(1-\frac{Q}{p}\right)}\left(\int_{X} \varrho^{p} d \mu\right)^{\frac{1}{p}} \leq C R^{1-\frac{Q}{p}}\left(\int_{X} \varrho^{p} d \mu\right)^{\frac{1}{p}} .
\end{aligned}
$$

To ensure that the above sum is finite we need the assumption that $p>Q$. As observed in Remark 4.4 this assumption is also necessary. The above estimate implies that

$$
\int_{X} \varrho^{p} d \mu \geq \frac{1}{C} \frac{R^{Q}}{R^{p}} \geq \frac{1}{C} \frac{\mu\left(B\left(x_{0}, R\right)\right)}{R^{p}} .
$$

By the above two cases, for all $\varrho \in F(\Gamma(r, R))$,

$$
\int_{X} \varrho^{p} d \mu \geq \frac{1}{C} \frac{\mu\left(B\left(x_{0}, R\right)\right)}{R^{p}}
$$

Taking the infimum over all such $\varrho$, we get

$$
M_{p}(\Gamma(r, R)) \geq \frac{1}{C} \frac{\mu\left(B\left(x_{0}, R\right)\right)}{R^{p}} .
$$

Our discussion of lower bounds for the $p$-modulus will be complete if we handle the case $Q-1<p<Q$. This will be obtained using the following result of HeinonenKoskela [HK].

Theorem 4.6. (Theorem 5.9 in $[\mathrm{HK}])$ Suppose that $(X, \mu)$ is a doubling space where the lower mass bound in (4.1) holds for some $Q \geq 1$. Suppose further that $X$ admits a weak $(1, p)$-Poincaré inequality for some $1 \leq p \leq Q$. Let $E$ and $F$ be two compact subsets of a ball $B_{R}$ in $X$ and assume that, for some $Q \geq s \geq Q-p$ and $1 \geq \lambda>0$, we have

$$
\min \left\{\mathscr{H}_{\infty}^{s}(E), \mathscr{H}_{\infty}^{s}(F)\right\} \geq \lambda R^{s-Q} \mu\left(B_{R}\right)
$$


Then there is a constant $C \geq 1$, depending only on $s$ and on the data associated with $X$, so that

$$
\int_{B_{C R}} \varrho^{p} d \mu \geq \frac{1}{C} \lambda \mu\left(B_{R}\right) R^{-p},
$$

whenever $u$ is continuous function in the ball $B_{C R}$ with $u \mid E \leq 0$ and $u \mid F \geq 1$, and $\varrho$ is a weak upper gradient of $u$ in $B_{C R}$.

Here $\mathscr{H}_{\infty}^{s}(E)$ denotes the Hausdorff $s$-content of a set $E$. Recall that if $E$ is connected, then $\mathscr{H}_{\infty}^{1}(E) \approx \operatorname{diam} E$.

Proposition 4.7. Let $X$ be a $Q$-Ahlfors regular metric measure space that supports $(1, p)$-Poincaré inequality for some $p>1$ such that $Q-1 \leq p \leq Q$ and $Q \geq 1$. Let $E$ and $F$ be continua contained in a ball $B_{R} \subset X$. Then

$$
M_{p}(\Delta(E, F, X)) \geq \frac{1}{C} \frac{\min \{\operatorname{diam} E, \operatorname{diam} F\}}{R^{1+p-Q}},
$$

for $C$ a constant in Theorem 4.6.

Proof. Take $s=1$ and define

$$
\lambda=\min \left\{1, \frac{1}{C} \frac{\min \left\{\mathscr{H}_{\infty}^{1}(E), \mathscr{H}_{\infty}^{1}(F)\right\}}{R^{1-Q} \mu\left(B_{R}\right)}\right\}
$$

for given continua $E$ and $F$. If $\lambda=1$, then by the Ahlfors regularity,

$$
1 \leq \frac{1}{C} \frac{\min \left\{\mathscr{H}_{\infty}^{1}(E), \mathscr{H}_{\infty}^{1}(F)\right\}}{R^{1-Q} \mu\left(B_{R}\right)} \leq 2 C,
$$

and so

$$
\lambda \approx \frac{1}{C} \frac{\min \left\{\mathscr{H}_{\infty}^{1}(E), \mathscr{H}_{\infty}^{1}(F)\right\}}{R^{1-Q} \mu\left(B_{R}\right)} .
$$

An application of Theorem 4.6 together with Ahlfors regularity yields

$$
\int_{B_{C R}} \varrho^{p} d \mu \geq \frac{1}{C} \frac{\min \{\operatorname{diam} E, \operatorname{diam} F\}}{R^{1+p-Q}}
$$

and the theorem now follows from the fact that $M_{p}(\Delta(E, F, X))=\operatorname{Cont}_{-C_{p}}(E, F, X)$, see Theorem 1.1 in Section 1.

The immediate consequence is a $p$-Loewner type result for $Q-1<p \leq Q$ :

Corollary 4.8. Under the assumptions of Proposition 4.7 on $X$, let $E$ and $F$ be continua with $\min \{\operatorname{diam} E, \operatorname{diam} F\}>0$. If $Q-1<p \leq Q$, then

$$
M_{p}(\Delta(E, F, X))>0 \text {. }
$$

In order to have complete analogy with the case $p>Q$ we would like to find a lower bound for $M_{p}(\Gamma(r, R))$ when $1 \leq p \leq Q$.

Theorem 4.9. Let $X$ be $Q$-Ahlfors regular metric measure space supporting $(1, p)$-Poincaré inequality. Assume also that $1 \leq p \leq Q$. Then we have for $0<r<R$ such that $X \backslash B\left(x_{0}, 2 R\right) \neq \emptyset$,

$$
M_{p}(\Gamma(r, R)) \geq \frac{1}{C} \frac{r^{Q p}}{R^{Q p+p-Q}} .
$$


Indeed, we can replace the condition that $X \backslash B\left(x_{0}, 2 R\right)$ is non-empty with the condition that there is a point $y \in X$ with $d\left(x_{0}, y\right)=3 R / 2$.

Proof. Let $x_{0} \in X$ and $\varrho \in F(\Gamma(r, R))$ be such that $\varrho \geq \epsilon>0$ on $B\left(x_{0}, R\right) \backslash$ $\bar{B}\left(x_{0}, r\right)$. Also assume that $\varrho$ is l.s.c. Let $u: X \rightarrow \overline{\mathbf{R}}$ be given by

$$
u(z)=\min \left\{\inf _{\substack{\gamma \text { connecting } \\ X \backslash B\left(x_{0}, R\right) \text { to } z}} \int_{\gamma} \varrho d s, 1\right\} \text {. }
$$

Observe that as $\varrho \in F(\Gamma(r, R))$, we have $u \equiv 0$ on $X \backslash \bar{B}\left(x_{0}, R\right), u \equiv 1$ on $\bar{B}\left(x_{0}, r\right)$, and $0 \leq u \leq 1$.

Let $B=B\left(x_{0}, 2 R\right)$. Note that

$$
u_{B}=\frac{1}{\mu(B)} \int_{B} u d \mu \geq \frac{\mu\left(B\left(x_{0}, r\right)\right)}{\mu\left(B\left(x_{0}, 2 R\right)\right)} .
$$

Because $X \backslash B\left(x_{0}, 2 R\right) \neq \emptyset$, by the path-connectedness of $X$ (which follows from the Poincaré inequality) there exists $y \in B\left(x_{0}, 2 R\right) \backslash B\left(x_{0}, R\right)$ such that $d\left(x_{0}, y\right)=\frac{3}{2} R$, $B\left(y, \frac{R}{2}\right) \subset B\left(x_{0}, 2 R\right) \backslash B\left(x_{0}, R\right)$ and

$$
\frac{1}{C} \leq \frac{\mu\left(B\left(y, \frac{R}{2}\right)\right)}{\mu\left(B\left(x_{0}, R\right)\right)} \leq C .
$$

The definition of $u$, the choice of $y$, and the Ahlfors regularity imply

$$
f_{B}\left|u-u_{B}\right|^{p} d \mu \geq \frac{1}{\mu(B)} \int_{B\left(y, \frac{R}{2}\right)} u_{B}^{p} d \mu \geq \frac{\mu\left(B\left(y, \frac{R}{2}\right)\right) \mu\left(B\left(x_{0}, r\right)\right)^{p}}{\mu\left(B\left(x_{0}, 2 R\right)\right)^{1+p}} \geq \frac{1}{C} \frac{\mu\left(B\left(x_{0}, r\right)\right)^{p}}{\mu\left(B\left(x_{0}, R\right)\right)^{p}} .
$$

Hence by the $(1, p)$-Poincaré inequality,

$$
\frac{1}{C} \frac{\mu\left(B\left(x_{0}, r\right)\right)}{\mu\left(B\left(x_{0}, R\right)\right)} \leq C R\left(f_{B} \varrho^{p} d \mu\right)^{\frac{1}{p}} \leq \frac{C R}{\mu\left(B\left(x_{0}, R\right)\right)^{\frac{1}{p}}}\left(\int_{X} \varrho^{p} d \mu\right)^{\frac{1}{p}} .
$$

From this we get

$$
\int_{X} \varrho^{p} \mathrm{~d} \mu \geq \frac{1}{C R^{p}} \frac{\mu\left(B\left(x_{0}, r\right)\right)^{p}}{\mu\left(B\left(x_{0}, R\right)\right)^{p-1}} .
$$

Inequality (4.19) now follows by taking infimum over all such $\varrho$ and by applying the Ahlfors regularity condition.

Remark 4.10. Following Remark 3.2 we compare the estimates obtained in [GM] to estimates derived in Theorem 4.3 and in Theorem 4.9. In our setting and notation Theorem 3.2 in $[\mathrm{GM}]$ states that with constant $C=C(p, Q, \Omega)$ if $0<r<$ $R<R_{0}(\Omega)$, then

$\operatorname{Cap}_{p}\left(\overline{B\left(x_{0}, r\right)}, B\left(x_{0}, R\right)\right) \geq\left\{\begin{array}{lr}C\left(1-\frac{r}{R}\right)^{p(p-1) \frac{\mu\left(B\left(x_{0}, r\right)\right)}{r^{p}},} & 1<p<Q, \\ C \frac{\mu\left(B\left(x_{0}, r\right)\right)}{r^{p}}\left(1-\frac{r}{R}\right)^{Q(Q-1)}\left(\log \frac{R}{r}\right)^{1-Q}, & p=Q, \\ C \frac{\mu\left(B\left(x_{0}, r\right)\right)}{r^{p}}\left(1-\frac{r}{R}\right)^{p(p-1)}\left|(2 R)^{\frac{p-Q}{p-1}}-r^{\frac{p-Q}{p-1}}\right|^{1-p}, & p>Q .\end{array}\right.$

However, note that if $p>Q$ and $r$ is comparable to $R$ then (4.10) is stronger than the corresponding estimate in [GM], since (4.10) does not involve $r$ at all. Moreover, if $1 \leq p \leq Q$ then inequality (4.19) for the cases $r \approx R$ or $r / R \approx 0$ gives a more delicate lower estimate than the corresponding part of Theorem 3.2 in [GM]. 


\section{5. -Modulus of curves passing through the point and Riesz potentials}

In Theorem 3.1 we showed that if $X$ is a doubling metric measure space, then $M_{p}(\Gamma)=0$, for $1 \leq p \leq Q_{2}$, where $\Gamma$ stands for the collection of all non-constant curves passing through $x_{0} \in X$. If $p>Q_{2}$, then $M_{p}(\Gamma)=\infty$, see Remark 4.5. The purpose of this section is to show that for the weighted Riesz measure, the $p$-modulus of $\Gamma$ remains positive for $p \geq 1$.

Another motivation for our studies comes from Theorem 2 in Keith's paper $[\mathrm{K}]$. Let $(X, d, \mu)$ be a complete doubling metric measure space. The property of $X$ of supporting a $(1, p)$-Poincaré inequality with respect to compactly supported Lipschitz functions and their compactly supported Lipschitz upper gradients is equivalent to the property of existing a constant $C \geq 1$ such that

$$
M_{p}\left(\Gamma_{x y}, \mu_{x y}^{C}\right) \geq \frac{1}{C} \frac{1}{d(x, y)^{p-1}}
$$

for almost every pair of distinct points $x, y \in X$. Here $\mu_{x y}^{C}$ denotes the symmetric Riesz kernel of $\mu\left(d \mu_{x y}^{C}=d \nu_{x}+d \nu_{y}\right.$ in the notation below).

Let $(X, d, \mu)$ be a doubling measure space. Consider the Riesz potential of a nonnegative function $f$ on $X$ (see e.g. Chapter 9 in [He])

$$
I_{1, B}(f)(x)=\int_{B} \frac{f(z) d(x, z)}{\mu(B(x, d(x, z)))} d \mu(z) .
$$

For a given point $x \in X$ define the measure $\nu_{x}(z)$ as follows:

$$
d \nu_{x}(z)=\frac{d(x, z)}{\mu(B(x, d(x, z)))} d \mu(z)
$$

By [He, Theorem 9.5] we know that if $X$ supports a $(1, p)$-Poincaré inequality for some $1 \leq p<\infty$, then for all balls $B$ in $X$ and all bounded continuous functions $u$ on $B$ and for all upper gradients $\varrho$ of $u$,

$$
\left|u(x)-u_{B}\right| \leq C(\operatorname{diam} B)^{p-1} I_{1, B}\left(\varrho^{p}\right)(x),
$$

whenever $x \in \frac{1}{2} B$.

Theorem 5.1. Let $(X, d, \mu)$ be a doubling measure space supporting Poincaré inequality for some $1 \leq p<\infty$. For a given point $x_{0} \in X$ consider measure $\nu_{x_{0}}(z)$ defined as in (5.2). Then

$$
M_{p}^{x_{0}}(\Gamma(r, R)):=\inf _{\varrho \in F(\Gamma)} \int \varrho(z)^{p} d \nu_{x_{0}}(z)>C,
$$

where $0<r<R$ such that $X \backslash B\left(x_{0}, 2 R\right)$ is non-empty and the constant $C$ depends only on the constants associated with the doubling property and the Poincaré inequality.

Proof. Take $0<r<R$ and consider the family of curves $\Gamma(r, R)$. As in Theorem 4.3 we define $u: X \rightarrow \mathbf{R}$ by

$$
u(z)=\min \left\{\inf _{\substack{\gamma \text { connecting } \\ z \text { to } X \backslash B\left(x_{0}, R\right)}} \int_{\gamma} \varrho d s, 1\right\} .
$$

From [JJRRS, Corollary 1.10], the function $u$ is measurable. Observe that as $\varrho \in$ $F(\Gamma(r, R))$, we have $u \equiv 0$ on $X \backslash \bar{B}\left(x_{0}, R\right)$ and $u \equiv 1$ on $\bar{B}\left(x_{0}, r\right)$. Consider 
$y \in B\left(x_{0}, 2 R\right) \backslash B\left(x_{0}, R\right)$ such that $d\left(x_{0}, y\right)=\frac{3}{2} R$ and consider a family of balls defined inductively

$$
\begin{aligned}
B_{0} & =B\left(x_{0}, 2 d\left(x_{0}, y\right)\right), B_{1}=B\left(x_{0}, d\left(x_{0}, y\right)\right)=B\left(x_{0}, 3 R / 2\right), \\
B_{i} & =\frac{1}{2} B_{i-1} \text { for } i>1, B_{-1}=B(y, 3 R / 2), B_{-i}=\frac{1}{2} B_{-i+1} \text { for } i>1 .
\end{aligned}
$$

By construction $u_{B_{-i}} \equiv 0$, for $i \geq 3$. Then by the doubling property of the measure and the Poincaré inequality we get the following estimate.

$$
\begin{aligned}
1 & =\left|u\left(x_{0}\right)-u(y)\right| \leq \sum_{i \in \mathbf{Z}}\left|u_{B_{i}}-u_{B_{i+1}}\right| \\
& \leq C \sum_{i=0}^{\infty} f_{B_{i}}\left|u(z)-u_{B_{i}}\right| d z+\sum_{i \leq-1}\left|u_{B_{i}}-u_{B_{i+1}}\right| \\
& =C \sum_{i=0}^{\infty} f_{B_{i}}\left|u(z)-u_{B_{i}}\right| d z+\sum_{i=1}^{3}\left|u_{B_{-i}}-u_{B_{-i+1}}\right| \\
& \leq C \sum_{i=0}^{\infty} \operatorname{diam} B_{i}\left(f_{B_{i}} \varrho^{p} d z\right)^{\frac{1}{p}}+C \sum_{i=1}^{3} d\left(x_{0}, y\right) 2^{-i}\left(f_{B_{-i}} \varrho^{p} d z\right)^{\frac{1}{p}} .
\end{aligned}
$$

Next we apply the Ahlfors regularity and Definition 5.1 to the first term of (5.7).

$$
\begin{aligned}
& C \sum_{i=0}^{\infty} \operatorname{diam} B_{i} \mu\left(B_{i}\right)^{-\frac{1}{p}}\left(\int_{B_{i}} \frac{\varrho^{p} d\left(x_{0}, z\right)}{\mu\left(B\left(x_{0}, d\left(x_{0}, z\right)\right)\right)} \frac{\mu\left(B\left(x_{0}, d\left(x_{0}, z\right)\right)\right)}{d\left(x_{0}, z\right)} d z\right)^{\frac{1}{p}} \\
& \leq C \sum_{i=0}^{\infty} \operatorname{diam} B_{i} \mu\left(B_{i}\right)^{-\frac{1}{p}}\left(\int_{B_{i}} \frac{\varrho^{p} d\left(x_{0}, z\right)}{\mu\left(B\left(x_{0}, d\left(x_{0}, z\right)\right)\right)} d\left(x_{0}, z\right)^{Q_{2}-1} d z\right)^{\frac{1}{p}} \\
& \leq C \sum_{i=0}^{\infty}\left(3 \cdot 2^{-i+1} R\right)\left(3 \cdot 2^{-i+1} R\right)^{\frac{-Q_{2}}{p}}\left(3 \cdot 2^{-i+1} R\right)^{\frac{Q_{2}-1}{p}}\left(\int_{B_{i}} \frac{\varrho^{p} d\left(x_{0}, z\right)}{\left.\mu\left(x_{0}, d\left(x_{0}, z\right)\right)\right)} d z\right)^{\frac{1}{p}} \\
& \leq C R^{\frac{p-1}{p}} \sum_{i=0}^{\infty} 2^{-\frac{p-1}{p}}\left(\int_{X} \varrho^{p} d \nu_{x_{0}}\right)^{\frac{1}{p}} \leq C R^{\frac{p-1}{p}}\left(\int_{X} \varrho^{p} d \nu_{x_{0}}\right)^{\frac{1}{p}} .
\end{aligned}
$$

Similar approach applied to the second term of (5.7) gives the following:

$$
\sum_{i=1}^{3} d\left(x_{0}, y\right) 2^{-i}\left(f_{B_{-i}} \varrho^{p} d z\right)^{\frac{1}{p}} \leq 3 C R^{\frac{p-1}{p}}\left(\int_{X} \varrho^{p} d \nu_{x_{0}}\right)^{\frac{1}{p}} .
$$

Therefore, (5.8) and (5.9) result in the inequality:

$$
\frac{1}{C} R^{1-p} \leq \int_{X} \varrho^{p} d \nu_{x_{0}}
$$

Take $R=1$. The proof of Proposition 5.1 now follows from the fact that the modulus is an outer measure on the space of all curves in $X$, and so for every $0<r<1<R$ we have:

$$
M_{p}^{x_{0}}(\Gamma(r, R)) \geq M_{p}^{x_{0}}(\Gamma(r, 1)) \geq \frac{1}{C}
$$




\section{References}

[Ah] Ahlfors, L. V.: Lectures on quasiconformal mappings. - Univ. Lecture Ser. 38, Amer. Math. Soc., 2nd edition, 2006.

[C1] Caraman, P.: Relations between $p$-capacity and p-module (I). - Rev. Roumaine Math. Pures Appl. 39:6, 1994, 509-553.

[C2] Caraman, P.: Relations between $p$-capacity and $p$-module (II). - Rev. Roumaine Math. Pures Appl. 39:6, 1994, 555-577.

[C3] Caraman, P.: p-module for Grötzsch and Teichmüller rings. - Rev. Roumaine Math. Pures Appl. 39:6, 1994, 579-585.

[C4] Caraman, P.: Some critical remarks on the equality of $p$-module and $p$-capacity. - Rev. Roumaine Math. Pures Appl. 47:5-6, 2002, 621-644.

[GM] Garofalo, N., and N. Marola: Sharp capacitary estimates for rings in metric spaces. - Houston J. Math. 36, 2010, 681-695.

[Ge] Gehring, F. W.: Rings and quasiconformal mappings in space. - Trans. Amer. Math. Soc. 103, 1962, 353-393.

[He] Heinonen, J.: Lectures on analysis on metric spaces. - Springer-Verlag, New York, 2001.

[HK] Heinonen, J., and P. Koskela: Quasiconformal maps in metric spaces with controlled geometry. - Acta Math. 181, 1998, 1-61.

[HKM] Heinonen, J., T. Kilpeläinen, and O. Martio: Nonlinear potential theory of degenerate elliptic equations. - Dover Publications, 2006.

[JJRRS] JärvenpäÄ, E., M. JÄrvenpä̈̈, K. Rogovin, S. Rogovin, and N. Shanmugalingam: Measurability of equivalence classes and $\mathrm{MEC}_{p}$-property in metric spaces. - Rev. Mat. Iberoamericana 23:3, 2007, 811-830.

[KSh] Kallunki, S., and N. Shanmugalingam: Modulus and continuous capacity. - Ann. Acad. Sci. Fenn. Math. 26:2, 2001, 455-464.

[K] KeIth, S.: Modulus and the Poincaré inequality on metric measure spaces. - Math. Z. $245: 2,2003,255-292$.

[Ko] Korte, R.: Geometric implications of the Poincaré inequality. - Results Math. 50:1-2, 2007, 93-107.

[MV1] Martio, O., and J. VÄISÄLÄ: Elliptic equations and maps of bounded length distortion. - Math. Ann. 282:3, 1988, 423-443.

[MV2] Martio, O., and J. VÄIsÄLä: Bounded turning and passability. - Results Math. 24:3-4, 1993, 246-273.

[Nä] NÄkki, R.: Prime ends and quasiconformal mappings. - J. Analyse Math. 35, 1979, 13-40.

[Oh] Ohtsuka, M.: Dirichlet problem, extremal length and prime ends. - Van Nostrand Reinhold Company, 1970.

[Ru] Rudin, W.: Real and complex analysis. - McGraw-Hill, New York-Düsseldorf-Johannesburg, 2nd edition, 1974.

[Vä] VÄISÄLÄ, J.: Lectures on $n$-dimensional quasiconformal mappings. - Lecture Notes in Math. 229, Springer-Verlag, Berlin-Heidelberg-New York, 1971.

Received 20 November 2009 